\title{
C2absorption-line diagnostics of diffuse interstellar clouds
}

\section{Citation}

Cecchi-Pestellini, Cesare, and Alexander Dalgarno. 2002. "C2absorption-Line Diagnostics of Diffuse Interstellar Clouds." Monthly Notices of the Royal Astronomical Society 331 (3) (April): L31-L34. doi:10.1046/j.1365-8711.2002.05418.x.

\section{Published Version}

doi:10.1046/j.1365-8711.2002.05418.x

\section{Permanent link}

http://nrs.harvard.edu/urn-3:HUL.InstRepos:30403734

\section{Terms of Use}

This article was downloaded from Harvard University's DASH repository, and is made available under the terms and conditions applicable to Other Posted Material, as set forth at http:// nrs.harvard.edu/urn-3:HUL.InstRepos:dash.current.terms-of-use\#LAA

\section{Share Your Story}

The Harvard community has made this article openly available.

Please share how this access benefits you. Submit a story.

\section{Accessibility}




\title{
$\mathrm{C}_{2}$ absorption-line diagnostics of diffuse interstellar clouds
}

\author{
Cesare Cecchi-Pestellini ${ }^{1,2 \star}$ and Alexander Dalgarno ${ }^{1}$ \\ ${ }^{1}$ Harvard-Smithsonian Center for Astrophysics, 60 Garden Street, Cambridge, MA 02138, USA \\ ${ }^{2}$ Dipartimento di Astronomia e Scienza dello Spazio, Università di Firenze, Largo E. Fermi 2, 50125 Firenze, Italy
}

Accepted 2002 February 11. Received 2002 February 11; in original form 2002 January 14

\begin{abstract}
A B S T R A C T
The excitation of the diatomic carbon molecule in diffuse interstellar clouds is discussed for a cloud with large density fluctuations of small linear filling factor along the line of sight. Possible implications for the chemistry of the diffuse cloud in front of Cygnus OB2 No. 12 are discussed.
\end{abstract}

Key words: molecular processes - ISM: clouds - ISM: individual: Cygnus OB2 No. 12 ISM: molecules.

\section{INTRODUCTION}

$\mathrm{C}_{2}$ radicals play an important role in the study of the structure, properties and evolution of diffuse interstellar clouds. The homonuclear molecule $\mathrm{C}_{2}$ was originally thought to be an effective optical probe of kinetic temperatures in interstellar clouds (Souza $\&$ Lutz 1977). However, radiative pumping effects, similar to those affecting rotational populations in $\mathrm{H}_{2}$, have been shown to be competitive with the collisional processes (van Dishoeck \& Black 1982), reducing the value of $C_{2}$ as a direct probe of kinetic temperature but providing a new source of information on the radiation field and molecular processes that determine rotational excitation.

Several absorption lines of $\mathrm{C}_{2}$ arising from different rotational levels belonging to the ground electronic and vibrational state $\mathrm{X}^{1} \Sigma_{\mathrm{g}}^{+}, v=0$, mainly in the Phillips and Mulliken systems, have been observed in interstellar clouds. Interstellar $\mathrm{C}_{2}$ was discovered towards $\zeta$ Oph by Chaffee \& Lutz (1978) who detected one line of the 2-0 band of the Phillips system at $10148 \AA$. Snow (1978) reported a detection of a line of the $0-0$ Mulliken system at $2313 \AA$ in a Copernicus ultraviolet spectrum of the same object. The $0-0$ band has been studied in detail by Lambert, Sheffer \& Federman (1995), using a high-resolution spectrum of $\zeta$ Oph from the Hubble Space Telescope. They also detected the weaker $\mathrm{F}^{1} \Pi_{\mathrm{u}}-\mathrm{X}^{1} \Sigma_{\mathrm{g}}^{+} 0-0$ band at $1342 \AA$. The 3-0 Phillips band at $7720 \AA$, weaker by a factor of 2 than the 2-0 band, was discovered by van Dishoeck \& Black (1986).

The $\mathrm{C}_{2}$ molecule rotational ladder, in sharp contrast to those of heteronuclear molecules like $\mathrm{CN}$ or $\mathrm{CO}$, has a significant population of high- $J$ levels. The high- $J$ level populations are extremely sensitive to the ambient gas density, and if accurate column densities for levels $J>4$ are available, the gas density can be inferred. In the uniform-density model of van Dishoeck \& Black (1982), the distribution of the rotational levels is determined by the ratio $n_{\mathrm{c}} \sigma_{0} / I$, where $n_{\mathrm{c}}$ is the density of the collision partners, $\sigma_{0}$ is

^E-mail: ccecchi-pestellini@cfa.harvard.edu the cross-section for $J=2 \rightarrow 0$ quenching, and $I$ is the incident interstellar radiation field.

The observed rotational level distribution does not, however, exclude the presence of high-density gas as in the model of the cloud in front of Cyg OB2 No. 12 (Cecchi-Pestellini \& Dalgarno 2000). At the temperatures characteristic of interstellar clouds, an increase in density reduces significantly the populations of high rotational levels as the collisions drive them towards thermal equlibrium. Then if most of the column density of $\mathrm{C}_{2}$ in the lowlying $J$ levels along the line of sight resides in the diffuse gas, the high-density component may be present but undetectable. The $\mathrm{C}_{2}$ rotational distribution arising in a mixed-phase medium inferred by absorption measurements may mimic the response from a diffuse homogeneous gas at some average density and kinetic temperature.

In this Letter, we investigate the extent of the $\mathrm{C}_{2}$ rotational ladder perturbation arising from the presence of high-density regions randomly mixed with a more tenuous background medium. In Section 2 we briefly survey the molecular processes that govern the excitation of $\mathrm{C}_{2}$, while in Section 3 we present models of the equilibrium of $\mathrm{C}_{2}$ in diffuse interstellar clouds with embedded dense clumps. The results of the modelling procedure are applied to the line of sight towards Cyg OB2 No. 12 (Section 4), where Gredel, Black \& Yan (2001) observed a large number of $\mathrm{C}_{2}$ rotational lines. Recently, along this apparently diffuse line of sight, Geballe et al. (1999) detected the molecular ion $\mathrm{H}_{3}^{+}$with an abundance comparable to that found in dense clouds (Geballe \& Oka 1996). This detection has raised questions about the processes responsible for the production of such large amounts of $\mathrm{H}_{3}^{+}$in the diffuse gas, and about what physical conditions prevail in the medium (Geballe et al. 1999; Cecchi-Pestellini \& Dalgarno 2000; Gredel et al. 2001).

\section{THE C 2 MOLECULE}

In interstellar clouds, $\mathrm{C}_{2}$ molecules exist mainly in the $\mathrm{X}^{1} \Sigma_{\mathrm{g}}^{+}$state. The production of vibrationally and rotationally excited $\mathrm{C}_{2}$ is 
initiated by absorption of diffuse starlight in the allowed electronic transitions to the singlet $\mathrm{A}^{1} \Pi_{\mathrm{u}}$ (Phillips system), $\mathrm{D}^{1} \Sigma_{\mathrm{u}}^{+}$(Milliken system) and $F^{1} \Pi_{u}$ states. These absorptions are followed by fluorescence to the electronic ground state. Collisions with the ambient gas species will contribute to establish a steady-state distribution of level populations. During the fluorescence cascade any change $\Delta v$ in the vibrational quantum number is possible so that the molecule is left in an excited vibrational level in the $\mathrm{X}^{1} \Sigma_{\mathrm{g}}^{+}$ state. Because of the homonuclear nature of the molecule, no electric dipole transitions among vibrational or rotational levels are allowed within an electronic state. The main de-excitation processes are singlet-triplet (intercombination) transitions to levels of the $\mathrm{a}^{3} \Pi$ state, followed by transitions back to the $\mathrm{X}^{1} \Sigma_{\mathrm{g}}^{+}$ state. These intercombination transitions cool down the rotational and vibrational Boltzmann temperatures of the $\mathrm{C}_{2}$ radicals. This process dominates direct quadrupole transitions when $v \neq 0$ (Le Bourlot \& Roueff 1986).

We solve the balance equations by means of the cascade efficiency formalism (Black \& Dalgarno 1976) in the form given by Le Bourlot, Roueff \& Viala (1987). The final system for the fractional population of the first 10 rotational states $\left(J_{\max }=18\right)$ of the ground vibrational state, $f_{J}$, reads

$$
\begin{aligned}
& f_{J}\left(\sum_{J^{\prime} \neq J} C_{J, J^{\prime}} n_{\mathrm{H}_{2}}+A_{J, J-2}+\sum_{k \in \mathrm{A}, \mathrm{D}} \zeta_{J, k}\right) \\
& =\sum_{J^{\prime} \neq J} f_{J^{\prime}} C_{J^{\prime}, J} n_{\mathrm{H}_{2}}+f_{J+2} A_{J+2, J}+\sum_{J^{\prime}} f_{J^{\prime}} \sum_{k \in \mathrm{A}, \mathrm{D}} \zeta_{J^{\prime}, k} D_{k, J},
\end{aligned}
$$

where $C_{J, J^{\prime}}$ are the (de)excitation rates for $\mathrm{H}_{2}$ collisions, $A_{J, J-2}$ the (quadrupole) rotational transition probabilities (van Dishoeck \& Black 1982), and $\zeta_{J, k}$ the total radiative pumping out of the state $J$ to the vibro-rotational levels of the excited electronic states $A^{1} \Pi_{u}$ and $\mathrm{D}^{1} \Sigma_{\mathrm{u}}^{+}, \mathrm{A}$ and $\mathrm{D}$ respectively,

$\zeta_{J, k}=\frac{\pi e^{2}}{m c} \mathrm{f}_{J, k} I\left(\nu_{k, J}\right) \mathcal{X}$.

In this equation $I\left(v_{k, J}\right)$ is the interstellar radiation field at the transition frequency, $\mathcal{X}$ is the radiation field scaling factor, and $\mathrm{f}_{J, k}$

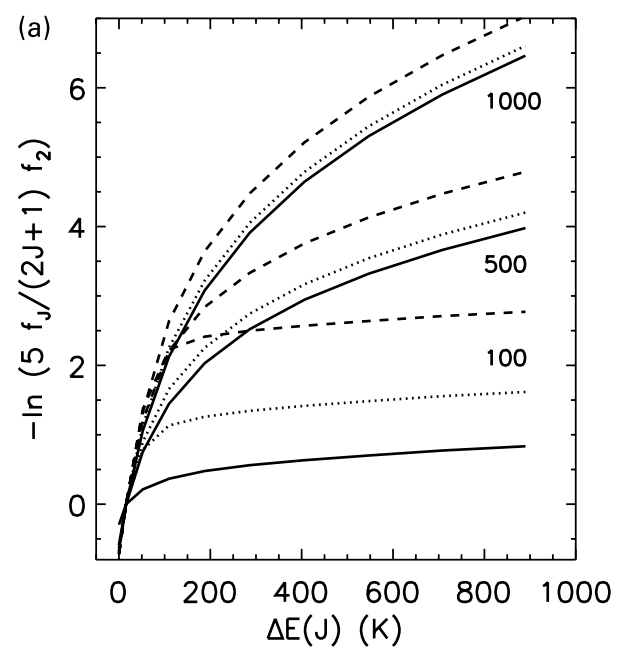

is the oscillator strength

$\mathrm{f}_{J, k}=1.499 \frac{2 k+1}{2 J+1}\left(\frac{\nu_{k, J}}{\mathrm{~cm}^{-1}}\right)^{2} A_{k, J}$,

where $A_{k, J}$ is the radiative transition probability. $D_{k, J}$ is the probability that a cascade from $\mathrm{A}^{1} \Pi_{\mathrm{u}}$ or $\mathrm{D}^{1} \Sigma_{\mathrm{u}}^{+}$leads to $\mathrm{X}^{1} \Sigma_{\mathrm{g}}^{+}(0, J)$ via all possible paths. Its definition is given by Le Bourlot et al. (1987). The fractional level populations are subject to the normalization condition $\sum_{i} f_{i}=1$.

References to the molecular constants are given by van Dishoeck \& Black (1982), Le Bourlot et al. (1987) and Rousselot et al. (2000).

\section{EQUILIBRIUM OF $\mathrm{C}_{2}$}

As discussed by van Dishoeck \& Black (1982), the observed $C_{2}$ rotational population distribution provides information on the temperature and density along the line of sight. The gas kinetic temperature, $T_{\mathrm{k}}$, is usually determined from the rotational excitation temperature, $T_{\mathrm{ex}}$, of the lowest rotational levels, because the population density in these levels is not significantly affected by radiative decays. Rotational diagrams are constructed with the level populations normalized to the population of the level $J=2$, forcing agreement for its column density, $N_{2}$ (cf. van Dishoeck \& Black 1982).

If dense gas is present, the measured column density of $\mathrm{C}_{2}$ in a rotational level $J$ is the superposition of the contributions from both the diffuse and dense phases, $\left(N_{J}\right)_{\mathrm{obs}}=N_{\mathrm{C}_{2}} f_{J}+N_{\mathrm{C}_{2}}^{*} f_{J}^{*}=$ $N_{J}\left(1+\eta f_{J}^{*} / f_{J}\right)$, where the superscript $*$ indicates quantities in local thermal equilibrium (LTE) and $\eta=N_{\mathrm{C}_{2}}^{*} / N_{\mathrm{C}_{2}}$ is the ratio between the total $\mathrm{C}_{2}$ column densities of the dense and diffuse phases. The relative populations as ratios of column densities are then given by

$$
\left(\frac{N_{J}}{N_{2}}\right)_{\mathrm{obs}}=\left(\frac{N_{J}}{N_{2}}\right) \frac{1+\eta f_{J}^{*} / f_{J}}{1+\eta f_{2}^{*} / f_{2}} .
$$

When $\eta=0$ we recover the ratio for homogeneous diffuse clouds.

We have computed the relative populations of the $\mathrm{C}_{2}$ rotational ladder (equation 4) as functions of the excitation energy $\Delta E(J)$ of level $J$ for several assumptions about the gas physical state. In Fig. 1 we show the results for two kinetic temperatures, $T_{\mathrm{k}}=20$ and

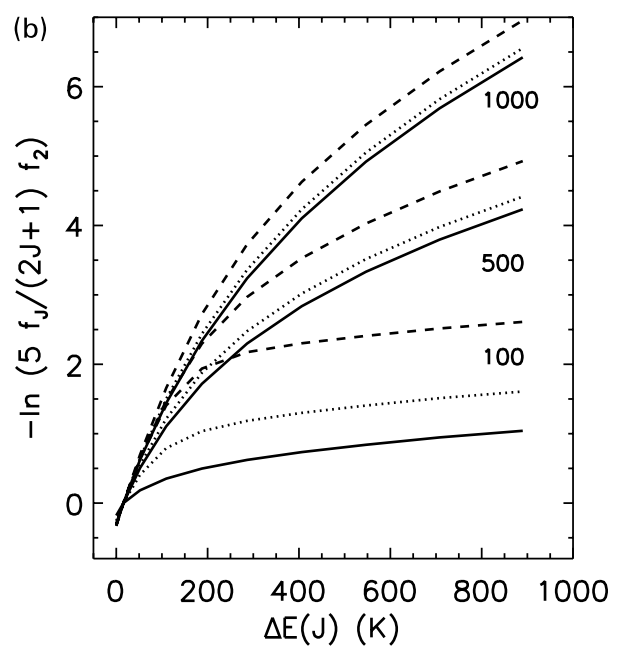

Figure 1. Relative rotational populations as functions of excitation energy $\Delta E(J)$. The distributions are shown for gas densities of 100,500 and $1000 \mathrm{~cm}^{-3}$. Solid lines: $\eta=0$; dotted lines: $\eta=0.1$; dashed lines: $\eta=0.5$. (a) $T_{\mathrm{k}}=20 \mathrm{~K}$; (b) $T_{\mathrm{k}}=45 \mathrm{~K}$. 


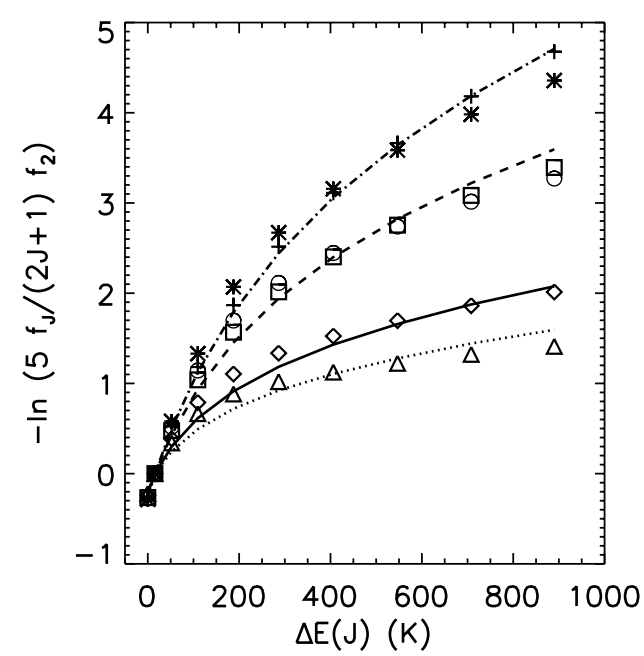

Figure 2. Relative rotational populations as functions of excitation energy $\Delta E(J)$ at $T_{\mathrm{k}}=50 \mathrm{~K}$. Dotted line: $n_{\mathrm{H}}=150 \mathrm{~cm}^{-3}$; solid line: $n_{\mathrm{H}}=$ $200 \mathrm{~cm}^{-3}$; dashed line: $n_{\mathrm{H}}=400 \mathrm{~cm}^{-3}$; dot-dashed line: $n_{\mathrm{H}}=600 \mathrm{~cm}^{-3}$. Points represent mixed-phase models - triangles: $n_{\mathrm{H}}=90 \mathrm{~cm}^{-3}, \eta=0.1$; diamonds: $n_{\mathrm{H}}=150 \mathrm{~cm}^{-3}, \eta=0.1$; squares: $n_{\mathrm{H}}=320 \mathrm{~cm}^{-3}, \eta=0.15$; circles: $n_{\mathrm{H}}=260 \mathrm{~cm}^{-3}, \eta=0.3$; asterisks: $n_{\mathrm{H}}=400 \mathrm{~cm}^{-3}, \eta=0.5$; pluses: $n_{\mathrm{H}}=560 \mathrm{~cm}^{-3}, \eta=0.1$.

$45 \mathrm{~K}$, and three different gas densities, $n_{\mathrm{H}}=100,500$ and $1000 \mathrm{~cm}^{-3}$. The assumed interstellar radiation field is given by van Dishoeck \& Black (1982). We set $\mathcal{X}=1$. The fractions of gas in LTE that we consider are $\eta=0,0.1$ and 0.5 . As expected, the changes introduced in the rotational distribution by the presence of packets of dense gas along the line of sight increase with decreasing density of the diffuse component. At low densities, $n_{\mathrm{H}} \leq 100 \mathrm{~cm}^{-3}$, and temperatures, $T_{\mathrm{k}} \leq 20 \mathrm{~K}$, intermediate rotational levels $(4 \leq J \leq 8)$ are considerably underpopulated with respect to LTE. Thus the excitation temperatures reflect the presence of very small fractions of dense gas, as low as a few per cent along the line of sight. For larger densities of the dominant diffuse component, $\mathrm{C}_{2}$ rotational levels are insensitive to density fluctuations of relatively small linear filling factor.

The average excitation temperatures derived from homogeneous models can be obtained from mixed-phase descriptions with suitable kinetic temperatures and densities of the two phases. The rotational diagrams corresponding to constant low-density models are well reproduced by two-phase models with a range of linear filling factors for the dense component and densities of the diffuse background (Fig. 2). Departures from the relative population ratios derived from the standard homogeneous model are very small and well inside the characteristic observational uncertainties. In all models the fraction of dense gas exceeds $\eta=0.1$. Thus $\mathrm{C}_{2}$ absorption-line diagnostics appear unable to detect the presence of small-scale density fluctuations, unless the gas along the line of sight has a low density $n_{\mathrm{H}} \leq 100 \mathrm{~cm}^{-3}$ and a low temperature $T_{\mathrm{k}} \leq 20 \mathrm{~K}$.

\section{THE CASE OF CYGNUS OB 2 NO. 12}

The recent discovery of $\mathrm{H}_{3}^{+}$towards the heavily reddened star Cygnus OB2 No. 12 (Geballe et al. 1999) has renewed interest in the diffuse cloud in front of the star. Geballe et al. (1999) constructed a model of the line of sight towards Cyg OB2 No. 12 with a hydrogen density of $10 \mathrm{~cm}^{-3}$ that, as they noted, led to an excessively long path length and did not explain the observations of

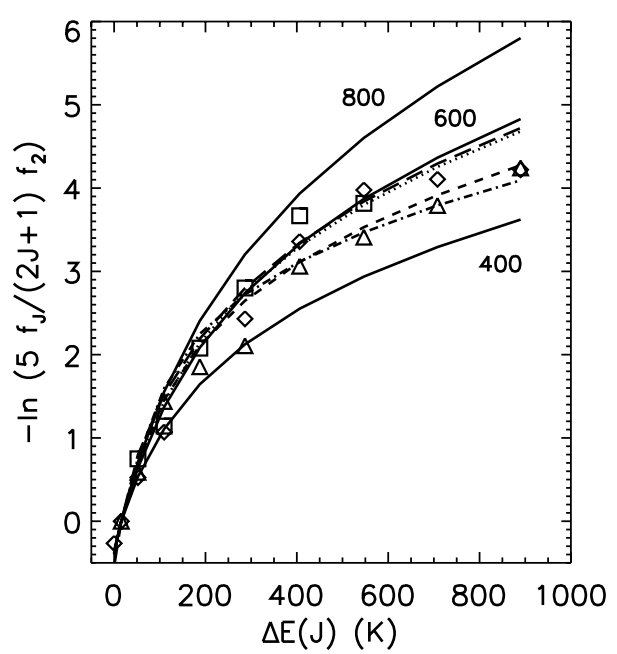

Figure 3. $\mathrm{C}_{2}$ excitation diagram of Cyg OB2 No. 12. The three solid lines drawn in the diagram represent model population distributions at $T_{\mathrm{k}}=35 \mathrm{~K}$ and $n_{\mathrm{H}}=400,600$ and $800 \mathrm{~cm}^{-3}$. Points correspond to individual line detections in the $(2-0)$ band (triangles: $\mathrm{R}$ branch; diamonds: $\mathrm{Q}$ branch; squares: P branch). Dotted line: $n_{\mathrm{H}}=540 \mathrm{~cm}^{-3}, \eta=0.1$; dashed line: $n_{\mathrm{H}}=440 \mathrm{~cm}^{-3}, \eta=0.2$; dot-dashed line: $n_{\mathrm{H}}=300 \mathrm{~cm}^{-3}, \eta=0.4$; longdashed line: $n_{\mathrm{H}}=540 \mathrm{~cm}^{-3}, \eta=0.1, T_{\mathrm{k}}=15 \mathrm{~K}$ in the dense phase.

CO (2-1) emission (Geballe et al. 1999) and $\mathrm{C}_{2}$ absorption (Gredel \& Münch 1994). The low-density model of Geballe et al. (1999) is consistent with the ISO observations that have shown the presence of the 3.4- $\mu \mathrm{m}$ hydrocarbon feature, and the absence of the 3.0- and 4.27- $\mu \mathrm{m}$ features of $\mathrm{H}_{2} \mathrm{O}$ and $\mathrm{CO}_{2}$ ices, respectively (Whittet et al. 1997). The model was questioned by Cecchi-Pestellini \& Dalgarno (2000) because of the assumption that the fractional abundance of $\mathrm{H}_{2}$ was 0.25 , and they presented a model of the material in front of the star Cyg OB2 No. 12 in which dense cold cores are proposed to exist embedded in diffuse clumps of gas. The model is consistent with ISO data and the absence of ice features. It predicts the presence of $\mathrm{HCO}^{+}$in a detectable amount. The recent detection of $\mathrm{HCO}^{+}(1-0)$ emission along this line of sight by Scappini et al. (2000) provides support for this scenario. Yet another model was put forward by Gredel et al. (2001) in which the molecular abundances are a response to an $\mathrm{X}$-ray ionization rate $\zeta \approx 10^{-15} \mathrm{~s}^{-1}$.

To constrain the physical conditions of density and temperature of the cloud molecular material in front of Cyg OB2 No. 12, Gredel et al. (2001) performed a detailed analysis of $\mathrm{C}_{2}$ interstellar absorption lines up to $J=18$ in the $(2,0)$ and $(3,0)$ bands of the Phillips system. The large number of rotational lines constrains the gas kinetic temperature and the density to $T_{\mathrm{k}}=35 \mathrm{~K}$ and $n_{\mathrm{H}}=600 \pm 100 \mathrm{~cm}^{-3}$. These conditions do not exclude the clumpy model of Cecchi-Pestellini \& Dalgarno (2000). In Fig. 3 we show some theoretical population distributions computed for a temperature of $T_{\mathrm{k}}=35 \mathrm{~K}$ using both homogeneous and mixedphase models. Only the $(2-0)$ band rotational transitions are shown. We did not perform any averages for individual rotational levels. The spread in the populations arising from different branches may be taken as a rough indication of the measurement errors. Assuming the distribution profile derived from a homogeneous cloud at $n_{\mathrm{H}}=600 \mathrm{~cm}^{-3}$ as the best fit to the observations (Gredel et al. 2001), we find that the mixed-phase model with $n_{\mathrm{H}}=540 \mathrm{~cm}^{-3}$ and $\eta=0.1$ is in excellent agreement with the data. More extreme models with $\eta=0.2$ and 0.4 fit the observations moderately well. Since temperature variations are 
likely to follow density fluctuations, we have also computed the level distribution profile assuming a lower kinetic temperature, $T_{\mathrm{k}}=15 \mathrm{~K}$, in the dense phase. We find no significant differences.

These results imply that the observed $\mathrm{C}_{2}$ rotational distribution is not inconsistent with the presence of high-density gas of linear filling factor $\eta \leq 0.1$ along the line of sight. The above upper limit is derived assuming a similar $\mathrm{C}_{2}$ fractional abundance in both diffuse and dense phases, and it could be larger because, according to Gredel \& Münch (1994), chemical models predict a sudden decrease in $\mathrm{C}_{2}$ abundance at densities above $10^{3.5} \mathrm{~cm}^{-3}$. Since the total hydrogen column density along the line of sight is $N_{\mathrm{H}}=$ $2 \times 10^{22} \mathrm{~cm}^{-2}$ (Geballe et al. 1999), the fraction of thermal gas yields $N_{\mathrm{H}}^{*} \leq 2 \times 10^{21} \mathrm{~cm}^{-2}$. Assuming in the high-density regime an almost total conversion of $\mathrm{C}$ to $\mathrm{CO}$, and adopting a carbon cosmic abundance of $1 \times 10^{-4}$ (Sofia, Fitzpatrick \& Meyer 1998), we obtain an upper limit for the CO column density produced in the dense phase of $N_{\mathrm{CO}}=2 \times 10^{17} \mathrm{~cm}^{-2}$, consistent with the observed value of $3 \times 10^{16} \mathrm{~cm}^{-2}$ (Geballe et al. 1999). Indeed, observations of the CO (2-1) emission by Scappini et al. (2000) imply a gas density $n_{\mathrm{H}} \geq 10^{4} \mathrm{~cm}^{-3}$.

\section{CONCLUSION}

We have shown that $\mathrm{C}_{2}$ absorption observations are not sensitive to the presence of dense gas of small linear filling factor along the line of sight. This result has implications for our understanding of the elusive chemistry of diffuse interstellar clouds. Millimetre-wave absorption spectra of molecules like CO, CS, SO, CN, HCN, HNC, $\mathrm{H}_{2} \mathrm{~S}, \mathrm{C}_{2} \mathrm{H}$ and $\mathrm{H}_{2} \mathrm{CO}$ (Lucas \& Liszt 1999) suggest large concentrations of these species in environments that are poorly shielded from the ambient ultraviolet radiation. In conventional chemical evolution models the physical characteristics of interstellar diffuse clouds are assumed to vary slowly on subparsec scales. While the clumpy structure of dense molecular clouds is widely accepted, the diffuse interstellar medium is usually considered a more homogeneous environment. The incorporation of density fluctuations, length-scale and filling factor in theoretical models could be crucial for the interpretation of diffuse cloud chemistry.

The chemistry of the cloud in front of Cyg OB2 No. 12 may be explained by a model in which dense cores are embedded in diffuse clumps of gas (Cecchi-Pestellini \& Dalgarno 2000). We have shown that $\mathrm{C}_{2}$ absorption measurements are consistent with this scenario. Since the $\mathrm{H}_{3}^{+}$molecular ion has now been definitely detected in at least four diffuse lines of sight (Geballe 2000), the chemistry of the gas is due not to the peculiarities of a particular region, but to a general feature of the diffuse interstellar medium.

Excitation analyses of other molecules in the framework of the mixed-phase model would be instructive.

\section{ACKNOWLEDGMENTS}

This research has been supported by the Division of Astronomy of the US National Science Foundation. CC-P thanks the Italian MURST.

\section{REFERENCES}

Black J. H., Dalgarno A., 1976, ApJ, 203, 132

Cecchi-Pestellini C., Dalgarno A., 2000, MNRAS, 317, L6

Chaffee F. H., Lutz B. L., 1978, ApJ, 221, L91

Geballe T. R., 2000, Phil. Trans. R. Soc. A, 358, 2503

Geballe T. R., Oka T., 1996, Nat, 384, 334

Geballe T. R., McCall B. J., Hinkle K. H., Oka T., 1999, ApJ, 510, 251

Gredel R., Münch G., 1994, A\&A, 285, 640

Gredel R., Black J. H., Yan M., 2001, A\&A, 375, 553

Lambert D. L., Sheffer Y., Federman S. R., 1995, ApJ, 438, 740

Le Bourlot J., Roueff E., 1986, J. Mol Spectrosc., 120, 157

Le Bourlot J., Roueff E., Viala Y., 1987, A\&A, 188, 137

Lucas R., Liszt H. S., 1999, in Ossenkopf V., Stutzki J., Winnewisser G., eds, The Physics and the Chemistry of the Interstellar Medium. GCAVerlag, Herdecke, p. 112

Rousselot P., Hill S. M., Burger M. H., Brain D. A., Laffont C., Moreels G., 2000, Icarus, 146, 263

Scappini F., Cecchi-Pestellini C., Codella C., Dalgarno A., 2000, MNRAS, 317, L6

Snow T. P., 1978, ApJ, 220, L93

Sofia U. J., Fitzpatrick E., Meyer D. M., 1998, ApJ, 499, 951

Souza S. P., Lutz B. L., 1977, ApJ, 216, L49

van Dishoeck E. F., Black J. H., 1982, ApJ, 258, 533

van Dishoeck E. F., Black J. H., 1986, ApJ, 307, 332

Whittet D. C. B. et al., 1997, ApJ, 490, 729

This paper has been typeset from a $\mathrm{T}_{\mathrm{E}} \mathrm{X} / \mathrm{L} \mathrm{T} \mathrm{E} \mathrm{X}$ file prepared by the author. 